\title{
Rasgos del semblante docente y su impacto en el aprendizaje: Una mirada desde la subjetividad del alumno
}

\section{Teacher's Appearance and its Effect on Learning: Student's Perspective}

\author{
Verónica Hernández Jacobo ${ }^{1}$ \\ Facultad de Psicología, \\ Universidad Autónoma de Sinaloa \\ México \\ hjvero2@gmail.com \\ Carlos Varela Nájera ${ }^{2}$ \\ Facultad de Psicología \\ Universidad Autónoma de Sinaloa \\ México \\ carlosvarela85@hotmail.com
}

Recibido 13 de octubre de 2011 • Aceptado 23 de febrero 2012

Resumen. Este trabajo forma parte de una investigación más amplia que analiza aquellos rasgos del semblante docente que promueven o inhiben el deseo de aprendizaje de estudiante. Tiene como referente teórico el psicoanálisis y los datos empíricos de estudiantes del grupo 602, de la Facultad de Psicología de la Universidad Autónoma de Sinaloa (UAS), en México, del ciclo escolar 2009-2010. Se analizan solo las características o rasgos del semblante que promueven el deseo de aprender de los estudiantes. El objetivo es conocer si el deseo de los estudiantes y las estudiantes de Psicología por aprender los contenidos de una asignatura es despertado cuando estos se identifican con el semblante de su docente, desde la premisa de que este posee rasgos o cualidades que permiten al estudiante identificarse con el llamado semblante docente. Se utiliza el enfoque cualitativo y el método fenomenológico. Las técnicas de recolección de datos fueron el cuestionario y la entrevista en profundidad. La principal conclusión fue que un mismo docente no impacta de la misma manera a sus estudiantes.

Palabras claves. Semblante docente, deseo de aprendizaje, subjetividad.

1 Docente de la Facultad de Psicología en materias del área de Psicología Social, y doctorante de la Facultad de Ciencias de la Educación de la Universidad Autónoma de Sinaloa (UAS), México. Línea de investigación sobre psicoanálisis y educación. Licenciada en Psicología, también por la UAS.

2 Docente de la Facultad de Psicología de la Universidad Autónoma de Sinaloa, México, en las materias de Psicoterapia, Psicología del crimen, Psicología de las masas, entre otras. Doctor en Educación con línea de investigación de Psicoanálisis y Educación, titular del Proyecto UASPROMEP sobre Psicopatologías y sus efectos en el aprovechamiento escolar: Intervención con niños y niñas de primarias públicas de Culiacán, Sinaloa, México. 


\begin{abstract}
This work is part of a larger research project, which analyzes those characteristics of the teacher's appearance that promote or inhibit the wish of students to learn. This study is based on the theoretical framework of psychoanalysis and the empirical data collected from the group 602, of the 20092010 academic year, of the Facultad de Psicología de la Universidad Autónoma de Sinaloa [Faculty of Psychology of the University of Sinaloa], Mexico. The study analyzes only those teacher's characteristics that motivate students to learn. The objective is to know if the desire of Psychology students to learn a subject matter sparks when they feel identified with the teacher's characteristics, upon the assumption that the teacher has characteristics or qualities that help students to feel identified with the so called "semblante docente" (teacher's profile). A qualitative approach and the phenomenological method were used in this study. The techniques of data collection included a questionnaire and in-depth interviews. The main conclusion was that the same teacher does not have always the same effect on his/her students.
\end{abstract}

Keywords. Teacher's appearance, learning desire, subjectivity.

\title{
Introducción
}

La relación docente-alumno, problema de estudio de este trabajo, es analizada desde diversas disciplinas como Educación, Pedagogía, Psicología, Psicopedagogía, Psicoanálisis, entre otras. El interés de nuestra investigación es analizar el vínculo docente-alumno desde los referentes psicoanalíticos, particularmente aquellos que abordan los aportes del Psicoanálisis a la Pedagogía. El primer registro de la relación de ambos discursos, menciona Filloux (2008), data de 1908 en la conferencia de Ferenzi, uno de los primeros discípulos de Freud. Su tema fue "Psicoanálisis y Pedagogía" y criticó, en esta, el carácter represivo de la educación de la época, particularmente porque veía la pedagogía como generadora de neurosis.

En esa misma tesitura, Oscar Pfister (citado por Filloux, 2008) señalaba que una Pedagogía que tome en cuenta los descubrimientos del Psicoanálisis permitiría preparar mejor al niño para una vida no neurótica. El Psicoanálisis, dice Pfister, debe ayudar al trabajo de los pedagogos, con fines morales, para liberar de una esclavitud del inconsciente.

Freud citado en Filloux (2008) planteaba que la educación inhibía, prohibía y sojuzgaba, y esto trae como consecuencia la neurosis. Por lo tanto, la educación debe tener equilibrio entrela escila de dejar hacer y el caridbis de la prohibición.

A partir de esos referentes, entre Psicoanálisis y Pedagogía, en esta investigación se reflexiona sobre conocer si el deseo de los estudiantes de psicología por aprender los contenidos de una asignatura es despertado cuando estos se identifican con el semblante de su docente en el recinto universitario de la Facultad de Psicología de la Universidad Autónoma de Sinaloa en México; desde las categorías semblante docente y deseo de aprendizaje de los estudiantes, respectivamente.

Este estudio se orientó a partir del siguiente supuesto: El deseo de los estudiantes por aprender los contenidos de una asignatura es despertado cuando estos se identifican con el semblante de su maestro, por lo tanto, el proceso de aprendizaje se favorecerá si los alumnos tienen ante ellos una figura digna de identificación; en contraparte, los estudiantes verán socavado su deseo de aprender cuando tienen ante sí un semblante docente con el cual no se identifican por ser una figura que no despierta su deseo de aprender.

El análisis del vínculo intersubjetivo docente-alumno en la educación superior atiende a que dicho vínculo está permeado por resistencias psíquicas, concepciones de la vida, prejuicios que llevan a rechazar al otro. El supuesto de partida es que el docente posee rasgos, cualidades o características 
que en muchas ocasiones pueden contribuir para que el estudiante despierte su deseo de aprender; pero también, como se pudo constatar en los resultados de esta investigación, el interés o deseo de los estudiantes por aprender disminuye ante la presencia de un docente cuyo semblante es poco "carismático". Es decir, en el mejor de los casos el docente tiene un semblante que cumple la función de señuelo, permitiendo que el alumno quede atrapado, capturado, o incluso engañado por su maestro, para posibilitar de esta manera, un lazo que ancle al alumno con el saber. Entonces, se entiende que el maestro, con su semblante garante del supuesto saber, focaliza el interés del estudiante hacia los contenidos de una asignatura, establece condiciones propicias para el aprendizaje y promueve un punto de encuentro entre el deseo del estudiante y la formalidad de la vida institucional.

La comunicación docente-alumno es un flujo constante de informaciones más allá del efecto habla y escucha, o del simple intercambio de palabras. Es el lenguaje más la forma de expresar, es la manera de dirigir el mensaje tanto con la voz como con el cuerpo; los gestos y ademanes son otras maneras de comunicarse entre profesor y estudiante, en estas formas de comunicarse el maestro o el alumno responden con agresividad, cinismo, burla o, incluso de parte del docente, la amenaza latente con la susodicha calificación de fin de semestre.

Desde el Psicoanálisis existen investigaciones que analizan la relación entre docentealumno, dentro de ellas están las que tienen como categorías centrales la transferencia amorosa, la identificación, la representación e imagen docente, entre otras. En este caso el abordaje en cuanto a las categorías de análisis centrales son semblante docente y deseo de aprendizaje de los estudiantes, a efecto de identificar la dinámica subjetiva en la relación docente-alumno.

Bochar (2003), en un trabajo titulado El docente y su relación con el saber que imparte, llegó a la conclusión de que la transferencia entre el grupo con el docente y viceversa permite producir más y mejor al grupo. Hay un compromiso mayor del estudiante, cuanto más se implica el docente con el saber que imparte. En ese mismo sentido, Núñez (2003) sostiene que el vínculo educativo, si se juega bien, puede ser una plataforma de lanzamiento a lo nuevo, a lo por venir: si se instala en la paradoja de sujetar para permitir que cada cual se lance a sus propias búsquedas.

En contraparte, Bischoff (2008), en su investigación titulada Vicisitudes entre el vinculo profesor-alumno, analiza el aspecto negativo del profesor, poniendo énfasis en las relaciones de poder y maltrato entre profesores y alumnos en el nivel medio, y concluye que ciertas características de personalidad del docente provocan distintas alteraciones que influyen negativamente en la disposición para aprender, las cuales se expresan a través de diversos síntomas psicosomáticos, cognitivos y conductuales que generan repitencias, cambios de escuelas, deserción escolar y, en algunos casos, abandono permanente de todo el sistema educativo.

Con base en los referentes anteriores se puede sostener que la personalidad del docente es un elemento subjetivo que atraviesa la relación pedagógica, que en palabras de los estudiantes que formaron parte de la investigación de campo, existe un cierto tipo de docente que les motiva a estar en clase y estudiar, pero otros disminuyen su deseo de continuar con su proceso de aprendizaje. De lo anterior se derivaron las siguientes interrogantes de investigación:

1. ¿Cómo se da la relación entre semblante del docente y deseo de aprendizaje de los estudiantes de psicología de la UAS?

2. ¿De qué manera influye el semblante del docente de psicología en el deseo de aprendizaje de sus estudiantes? 
La búsqueda de respuesta a tales preguntas tuvo como propósito central conocer si el deseo de los estudiantes de Psicología por aprender los contenidos de una asignatura es despertado cuando se identifican con el semblante de su docente; así como, aportar elementos para el análisis del papel que desempeña el docente en los procesos de enseñanza y aprendizaje.

\section{Metodología}

La estrategia de investigación seguida se inscribe en el enfoque cualitativo, pues se describen y comprenden los significados producidos en la relación semblante docente y deseo de aprendizaje de los estudiantes. Este fenómeno está enmarcado en el terreno subjetivo y, por tanto, habría que describirlo mediante el método fenomenológico.

El criterio de inclusión de los sujetos fue el siguiente: estudiantes cuyo grado de identificación con una materia estuviera marcada por la figura del profesor. Se llevó a cabo el trabajo de campo con un grupo de la Facultad de Psicología de sexto semestre, durante los meses de febrero a mayo del ciclo escolar 2009-2010.

Inicialmente se aplicó un cuestionario abierto a los 36 alumnos y alumnas del grupo 602, mediante el cual se recuperó información de aspectos socioeconómicos, académicos y familiares. Con base en esa información se construyó una tipología de estudiantes, a partir de los siguientes criterios

- La escolaridad de los padres, con el propósito de tener referentes académicos de la familia.

- Los ingresos económicos de la familia por mes, con la finalidad de asociar el capital económico al desempeño académico.

- El número de materias reprobadas como estudiantes de Psicología, en este parámetro se consideraron a estudiantes con el mayor número de materias reprobadas, así como los que tenían una o dos, y a aquellos estudiantes con ninguna materia reprobada.

- El gusto que por una asignatura fuera adjudicado al profesor que la imparte. Este último criterio, con la finalidad de articular la relación entre semblante docente y deseo de aprendizaje de los estudiantes.

Se identificaron diez tipos de estudiantes y, según el criterio de representación, se incluyó a un estudiante de cada uno esos tipos.

Posteriormente se les aplicó una entrevista cualitativa abierta o a profundidad. Según Sierra (1998), esta permite recoger una gran cantidad de información de una manera más cercana y directa entre investigador y sujeto de investigación.

\section{Análisis de resultados}

En opinión de los estudiantes, participantes en el estudio, los principales rasgos del profesor que despiertan el gusto por una materia, son los siguientes: 


\section{a) Rasgos intelectuales del docente}

- Me gustaba como daba la clase la maestra.

- La maestra hizo pensar, analizar comprender.

- El maestro denotaba que conocía la materia.

- Las cátedras del profesor me atraían.

- El profesor muestraba dominio del tema.

- El profesor era fluido y con una memoria que se acordaba de todo, sin leer notas.

\section{b) Rasgos de personalidad del docente}

- El manejo de la clase y la forma de ser del maestro.

- Clases interesantes y dinámicas por la maestra.

- La manera en que se expresaba la maestra.

- El profesor era fácil de darse a entender.

- La forma en que la maestra daba la clase.

- La maestra hace interesante la materia.

- La forma de actuar y vestir del profesor.

Estas referencias empíricas ponen de manifiesto que la relación maestro-alumno está atravesada por cuestiones subjetivas que deben ser estudiadas desde diversas categorías conceptuales. En este caso, se estudian el semblante del profesor y el deseo de los estudiantes; en la jerga pedagógica estaríamos haciendo referencia a qué aspectos del docente influyen o son significativos para despertar el deseo de aprendizaje de los estudiantes.

A partir de las entrevistas a profundidad realizadas a 10 sujetos, se pudieron categorizar los rasgos docentes de la siguiente manera: psicológicos, físicos o aliño personal, intelectuales o académicos.

Los fragmentos de las entrevistas a estudiantes que hacen alusión a los rasgos psicológicos del docente se presentan a continuación:

"La maestra T era muy puntual, y el maestro F en segundo año se llevaba muy bien con todos y aceptaba sugerencias. En la carrera, la maestra A era agradable, fue una de las mejores maestras porque era accesible, comprensible, era estricta pero era de esas mujeres de las que todo el mundo estaba en su clase, era muy fácil de interactuar con ella, yo le entendía bien" (S2F). "Me han llamado la atención los maestros que se nota que están preparados, por ejemplo aquí en la carrera tuve un maestro en primer año que sabía mucho, en clase no leía en los libros o en notas, todo se lo sabía, también una profesora que era muy estricta, pero nos hacía aprender" (S6F). "En profesional el profe V era muy carismático siempre estaba sonriente en clase muy atento, pero la más significativa en mi carrera es la maestra A, en primer lugar es una persona muy amable, y le da buen trato a sus alumnos" (S7F).

Lo anterior muestra que el docente impacta al alumno no solo con lo que sabe, sino también con sus cualidades psicológicas, que, en su conjunto, deben propiciar el interés del alumno hacia el 
aprendizaje. Según Mannoni (1982), la principal tarea del docente y de la Pedagogía en general es garantizar el deseo de saber, para ello es indispensable que el alumno se identifique con su docente y se "apropie" inconscientemente de ciertos rasgos valiosos de este. En este sentido, hay estudiantes que repiten los conceptos que utiliza el docente con el cual se identifican, imitándolo hasta en los gestos, estudian contenidos con referencia al área del conocimiento del maestro admirado, acompañan a este en sus investigaciones, seminarios o círculos de estudio.

Lo anterior nos pone frente al fenómeno de identificación, concepto psicoanalítico que designa "el proceso por el cual un individuo se hace semejante a otro, en su totalidad o en parte" (Chemama y Vandermersch, 2004. p. 340).

Los rasgos del semblante docente que hacen alusión a características físicas son los siguientes:

"Una maestra de Matemáticas la recuerdo bien porque lo que le resaltaba más era la parte de sus nalgas eran muy ancha nosotros nunca entendimos por qué las tenía así, no era algo normal" (S10M). "Se me hace muy adecuada la forma en que viene vestido el maestro U, bien aliñado, perfumado, siempre sonriendo y en el grupo recordemos su atractivo" (S4F). "Me identificaba mucho con una maestra que se pintaba los labios muy rojo, era muy bonita, interesante, yo quería ser como ella cuando creciera" (S3F).

Desde nuestro posicionamiento teórico, el análisis de las entrevistas muestra que el docente ocupa un lugar como objeto admirado por parte de sus alumnos, en la medida en que sea capaz de cumplir la función de vivificar el deseo de saber de sus estudiantes. Por lo tanto, más allá del grado académico, la formación y su capacitación para la docencia, tendrá que arreglárselas cara a cara con sus alumnos para posibilitar el deseo de aprender.

La categoría de semblante nos permite analizar la importancia del papel de los docentes en el proceso de aprendizaje, siempre y cuando explotamos su dimensión de establecimiento del lazo social, ya que produce cierta empatía o lo que conocemos desde el psicoanálisis clásico como transferencia. La dimensión de semblante opera a nivel latente construyendo y enriqueciendo lo inesperado del currículo que antes se nombraba como currículum oculto. Por otro lado, hacer uso del semblante pudiera tener consecuencias negativas, si el docente no lo utiliza con ética, pues algunos académicos que manejan cierto saber semblantean o apantallan al otro bajo la investidura de sabelotodo, con este supuesto someten al estudiantado hacia fines que solo benefician al docente, porque debemos recordar que el término semblante también hace alusión a un enmascaramiento, faire semblant de: darse la apariencia de, hacer como si, sinónimo de fingir y de simular (Guerrero y Hopen, 1999).

Por otra parte, las características del semblante docente que hacen referencia a cuestiones académicas o intelectuales del profesor que permiten despertar el deseo de aprendizaje de los estudiantes son como sigue, según fragmentos de las entrevistas:

"En primaria tuve un maestro explicaba muy bien, nos enseñó mucho, todavía lo recuerdo. En secundaria había un profesor de Matemáticas al cual le entendíamos, con él aprendí Matemáticas. En lo profesional, en primer año me llamó mucho la atención el maestro V porque, era muy diferente a los demás, los demás, trataban de conocernos con técnicas y el maestro V no, el venía a dar clases, se preocupa porque el otro aprenda" (S3F). 
"Me han llamado la atención los maestros que se nota que están preparados, por ejemplo aquí en la carrera tuve un maestro en primer año que sabía mucho, en clase no leía en los libros o en notas, todo se lo sabía, también una profesora que era muy estricta, pero nos hacía aprender" (S6F).

"En la carrera solo ha sido muy significativo un profesor que ya está jubilado, es el que podría decir que me gustaría ser como él me interesaron siempre sus cátedras, por metódico y preparado" (S5M).

Cuando un docente es admirado decimos freudianamente que sus estudiantes se han identificado con él, y socráticamente han visto en él un brillo, una hermosura, un agalma, un objeto preciado del cual intentan tomar pedazos, de ahí la idea de que el docente puede transmitir su deseo de saber en sus estudiantes despertando en ellos su propio deseo.

Esto nos lleva a considerar lo dicho por Camps (2005, párr. 6) “ (...) uno enseña con lo que es [no] con lo que sabe (...)", por lo tanto, la tarea del docente implica saber algo de quién es ese estudiante al que se dirige con su acto. Estaríamos frente a la dimensión de la posición y deseo del educador. El profesional no es un mero observador, es un agente cuyo acto tiene consecuencias. Es decir, cualquier posición que el docente tome impactará a su estudiante, para ser alguien respetado o devaluado.

Desde el Psicoanálisis se plantea que:

En la relación maestro-alumno cada cual ocupa un lugar específico y los lugares no son intercambiables. [El docente debe] saber que [sic] lugar ocupa y cual [sic] es su función, y la función del educador no es hacer de madre ni padre sino introducir al niño en el gusto por el saber. Se ha de ver que [sic] es lo que se puede hacer y lo que no, donde [sic] están los límites de la intervención" (Camps, 2005, La posición de docente, párr. 4).

\section{Conclusión}

El semblante tiene que ver con un enmascaramiento, con algo que engaña, con una simulación, entonces ¿qué referentes empíricos se pueden rescatar en este estudio para darle importancia al semblante "engañoso" y su relación con el deseo de aprendizaje? En la respuesta a este interrogante encontramos semblantes docentes tales como el maestro U, el maestro V, la maestra A, que permitieron apuntalar el deseo de aprender de algunos estudiantes, ya que dichos docentes pueden producir un lazo social con el alumno que lo lleve a identificarse y emular ciertas actitudes e intereses académicos de su docente admirado y valorado.

Para que un docente sea admirado debe cumplir con ciertas características que muy pocas veces son conscientes o apreciables experimentalmente. Lo que sí se puede rescatar son algunos rasgos mínimos que el docente debe tener para que sea valorado, respetado y admirado por sus estudiantes, entre algunos podríamos citar los que mencionaron los sujetos de investigación, tales como: dominio del tema, puntual, agradable, bien vestido, que sea coherente con lo que dice y hace, 
que se vea que le interesan sus estudiantes, que no solo se presente a trabajar por "el cheque", entre otras cosas que permiten dar valor a un docente.

Hay excepciones en que un mismo profesor o profesora puede capturar, atrapar, o bien, en palabras de Carrizales (1991), seducir pedagógicamente a sus estudiantes debido a que su semblante está conformado con una serie de rasgos como dominio del tema, voz interesante, aliño personal, pasión por su profesión y por su asignatura, que en palabras de los mismos alumnos El maestro X se apasiona cuando da la clase, tiene una voz y un conocimiento que nos captura.

A su vez, podemos rescatar ciertas actitudes o rasgos del semblante docente que no permiten que el estudiante se identifique con un maestro cuya imagen está devaluada por ciertas características de incompetencia o extravagancia, lo cual da como resultado que el docente no tenga nada, ni siquiera un semblante que engañe y que funja como amarre entre el estudiante y el aprendizaje, en cambio, lo que aparece en escena son maestros que "lloran en clase para causar lastima", "que no saben y se dedican a poner a exponer a los alumnos", "escupen sus problemas personales", "comen con la boca abierta en clase y se les ven las bubis desparramadas", "nos dicen inútiles y nos ofenden", "faltan todo el semestre y al final nos piden trabajos de investigación en una semana y nunca dicen cómo hacerlos", entre comentarios recuperados en las entrevistas, que podríamos cuestionar acerca de ¿qué estudiante podría identificarse con docentes cuyo semblante no favorece el lazo-social necesario para que el estudiante tenga un aliciente para el aprendizaje, y más aún para identificarse como profesionista de la Psicología?

A modo de cierre podemos decir que el semblante de un docente no influye de la misma manera a todos sus estudiantes. Hay rasgos o cualidades de un mismo docente que para unos estudiantes promueve el deseo de aprender y para otros lo inhibe. Por ejemplo, una profesora o profesor muy metódico - que toma lista, que organiza el curso- puede provocar que los estudiantes que también lo son se identifiquen con sus profesores. En cambio ese mismo docente puede inhibir el deseo de aprendizaje en aquellos estudiantes que no son organizados y les aburren "los formalismos".

Como lo narraron en las entrevistas los estudiantes “(...) la maestra $\mathrm{M}$ es muy estricta, por eso me gusta porque yo soy muy organizada". Sin embargo ante esta misma maestra, otros y otras alumnas hicieron alusión a lo siguiente: “(...) la maestra $\mathrm{M}$ es excelente en el dominio del tema, pero casi todos le tenemos miedo y varios compañeros del salón toman la materia con otro profesor o prefieren hacer extraordinario. Nótese por lo tanto que los alumnos también necesitan que el docente posea otros rasgos que no solo sean intelectuales, aquellos que posibilitan "la confianza de participar en clase". 


\section{Referencias}

Bischoff, G. (10 de setiembre de 2008). Vicisitudes entre el vínculo profesor alumno [Foro en línea: Actores sociales y relaciones de poder en los procesos educativos]. Universidad de Flores Facultad de Psicología y Ciencias Sociales. Recuperado de http://es.scribd.com/doc/48766076/ VICISITUDES-ENTRE-EL-VINCULO-ENTRE-PROFESOR-Y-ALUMNO

Bochar, J. (agosto, 2003). El docente y su relación con el saber que imparte. Revista Querencia, 6. Recuperado de http://www.querencia.psico.edu.uy/revista_nro6/jaqueline_bochar.htm

Camps, M. D. (2005). El malestar en el docente, su posición y su deseo. Trabajo presentado en el Seminario del campo freudiano de Valencia, curso 204-2005, grupo de investigación: Pedagogía y Psicoanálisis. Recuperado de http://es.scribd.com/doc/55950160/El-Malestar-enEl-Docente

Carrizales, C. (1991). El filosofar de los profesores. Culiacán, México: Universidad Autónoma de Sinaloa.

Chemama, R. y Vandermersch, B. (2004). Diccionario del Psicoanálisis (2a ed.). Buenos Aires: Amorrortu.

Filloux, J.-C. (2008). Psicoanálisis y Pedagogía: Una consideración del inconsciente en el campo pedagógico. En M. Jiménez, y R. Páez (Comps). (2008) Deseo, saber y transferencia. Un acercamiento psicoanalítico a la educación (pp. 27-72) México: Siglo XXI.

Guerrero, O. y Hopen, C. (1999). Un semblant más semblant (que el verdadero) o traducir el Sens blanc. Association Lacanienne internationale. Recuperado de http://www.freud-lacan.com/ Champs_specialises/Lacan_Espagnol/Un_semblant_mas_semblant_que_el_verdadero_o_ traducir_el_Sens_blanc

Mannoni, O. (1982). Psicoanálisis y enseñanza, un comienzo que aún no termina. Madrid: Paidós.

Núñez, V. M. (2003). El vínculo educativo. En H. Tizio (Coord.). (2003). Reinventar el vínculo educativo: Aportaciones de la Pedagogía social y del Psicoanálisis (pp. 19-48). Barcelona: Gedisa.

Sierra, F. (1998). Función y sentido de la entrevista cualitativa en investigación social. En J. Galindo (Coord.) Técnicas de investigación en sociedad, cultura y comunicación (pp. 277-341). México: Addison Wesley Longman. 